\title{
Study of the morphology of semicrystalline poly(ethylene terephthalate) by hydrolysis etching
}

\author{
M. E. Cagiao and F. J. Baltá Calleja* \\ Instituto de Estructura de la Materia, CSIC, Serrano 119, 28006 Madrid, Spain \\ and C. Vanderdonckt and H. G. Zachmann \\ Institut für Technische und Makromolekulare Chemie, University of Hamburg, \\ Bundesstrasse 45, 2000 Hamburg 13, Germany \\ (Received 25 March 1992; revised 30 July 1992)
}

\begin{abstract}
Semicrystalline poly(ethylene terephthalate) was hydrolysed in water at $180^{\circ} \mathrm{C}$ under elevated pressure and subsequently treated with ethanol, following the etching process first developed by Miyagi and Wunderlich. The weight loss, the wide-angle X-ray scattering and the molecular weight were measured as a function of etching time. It was found that even at the end of the etching process not all the amorphous material could be removed by the hydrolysis treatment. By comparing the obtained results with those derived from an elaborate small-angle $X$-ray scattering study and with wide-angle $X$-ray scattering measurements, it was concluded that only those amorphous regions lying outside of the lamellar stacks were removed. Subsequently, the lamellar stacks themselves were attacked. It was also found that at the very beginning of the hydrolysis process additional crystals were formed in the material.
\end{abstract}

(Keywords: morphology; semicrystalline polymer; poly(ethylene terephthalate)

\section{INTRODUCTION}

Chemical etching followed by molecular weight measurements is a well established method for studying the morphology of semicrystalline polymers ${ }^{1,2}$. By subjecting the polymer to an appropriate chemically aggressive liquid, the chemical bonds of the chain molecules in the amorphous regions are broken. Subsequently, the amorphous regions can be removed by a solvent. It is assumed that, under ideal conditions, the material left in the sample consists of oligomers having a chain length equal to the thickness of the crystals. However, these ideal conditions are often not achieved ${ }^{3-5}$. Etching techniques using hydrolysis in water were first performed for the analysis of cellulose ${ }^{4}$ and later were also applied to polyamides ${ }^{5}$ and polyesters ${ }^{6,7}$. For the etching of polyolefins, oxidative attack with fuming nitric acid is selective enough to reveal the lamellar morphology of polyethylene ${ }^{2}$.

An extensive study of etching of poly(ethylene terephthalate) (PET) in pure water was performed by Miyagi and Wunderlich ${ }^{6,7}$. These authors showed that by exposing the sample to pure water under elevated pressure at $180^{\circ} \mathrm{C}$ followed by extraction in ethanol, a fraction of the material (up to $40 \%$ ) is removed. Simultaneously, the molecular weight $\left(M_{\mathrm{w}}\right)$ of the sample strongly decreased. Obviously, during the first process at $180^{\circ} \mathrm{C}$, part of the material was hydrolysed to ethylene

\footnotetext{
* To whom correspondence should be addressed
}

glycol and terephthalic acid which was then dissolved by the ethanol. In the further evaluation, Miyagi and Wunderlich concluded that the amorphous material was removed from the sample completely. This last contention, however, becomes questionable, because the degree of crystallinity was calculated from the density of the crystals, using the value $\rho_{\mathrm{c}}=1.445 \mathrm{~g} \mathrm{~cm}^{-3}$ which was given by Daubeny et $\mathrm{al}^{8}$. This value was later proved to be incorrect ${ }^{9-11}$ and was replaced by a higher value of $\sim 1.490 \mathrm{~g} \mathrm{~cm}^{-3}$. Unfortunately no wide-angle X-ray scattering (WAXS) measurements were performed to obtain more reliable information on the degree of crystallinity. Furthermore, in the small-angle X-ray scattering (SAXS) measurements, Miyagi and Wunderlich only evaluated the position of the intensity maximum by applying Bragg's law; more efficient methods can now be applied $^{12}$. Therefore, further investigations of this etching process using the improved value of $\rho_{\mathrm{c}}$ and including additional methods like WAXS and more elaborate SAXS analyses seem to be appropriate.

In preceding SAXS studies ${ }^{12}$, we have found that there exist two kinds of amorphous regions differing in size in PET crystallized as completely as possible. The aim of the present investigation is to apply the powerful etching method developed by Miyagi and Wunderlich to study the morphology of such a sample. The etching experiments are supplemented by other measurements like density, WAXS and microhardness. The results will be compared to those obtained in a recent study of the morphology by means of SAXS ${ }^{12}$. 


\section{EXPERIMENTAL}

PET was synthesized from dimethyl terephthalate in our laboratory using manganese acetate and $\mathrm{Sb}_{2} \mathrm{O}_{3}$ as catalysts as described earlier ${ }^{13}$. The molecular weight $\left(M_{\mathrm{w}}\right)$, as measured by viscometry in hexafluoroisopropanol ${ }^{14}$, using the equation:

$$
[\eta]=5.2 \times 10^{-4} M_{\mathrm{w}}^{0.695}
$$

was 18000 .

Amorphous films (200-250 $\mu$ m thick) were obtained by melt pressing in vacuo at $280^{\circ} \mathrm{C}$ for 10 min and quenching in ice-water. The starting materials were first crystallized at $240^{\circ} \mathrm{C}$ for $24 \mathrm{~h}$ in vacuo and then cut into very small pieces $(\sim 1 \times 1 \mathrm{~mm})$. Then, $\sim 0.5 \mathrm{~g}$ of the crystallized sample was put into a glass tube with $1 \mathrm{~cm}^{3}$ of water. The glass tubes were $8 \mathrm{~mm}$ in diameter and their inner volume was $\sim 10 \mathrm{ml}$. After air evacuation, the glass tubes were sealed and hydrolysis was carried out at $180^{\circ} \mathrm{C}$ at elevated pressure for different times up to $122 \mathrm{~h}$. The soluble part of the sample was finally extracted by ethanol using a Soxhlet.

The density $(\rho)$ was measured in a density gradient column filled with hexane/tetrachloroethane. The degree of crystallinity $\left(x_{c}\right)$ was calculated by means of the equation $^{11}$ :

$$
x_{\mathrm{c}}=\left(\rho_{\mathrm{c}} / \rho\right)\left(\rho-\rho_{\mathrm{a}}\right) /\left(\rho_{\mathrm{c}}-\rho_{\mathrm{a}}\right)
$$

with $\rho_{\mathrm{c}}=1.490 \mathrm{~g} \mathrm{~cm}^{-3}$ and $\rho_{\mathrm{a}}=1.334 \mathrm{~g} \mathrm{~cm}^{-3}$.

The WAXS diagrams were measured in the transmission mode using a Siemens D-500 goniometer. After subtraction of the air and Compton scattering, the amorphous halo was fitted to the curve at small scattering angles as for the fits performed in a previous paper ${ }^{11}$ based on the method developed by Ruland ${ }^{15,16}$.

The microhardness $(H)$ was determined at room temperature using a Leitz tester with a square-based diamond indenter ${ }^{17}$. The $H$ value was calculated from the residual projected diagonal impression. A loading cycle of $0.1 \mathrm{~min}$ to minimize creep of the material under the indenter was adopted.

\section{RESULTS}

Figure 1 shows the fraction of material removed by hydrolysis $\left(x_{\mathrm{H}}\right)$ as a function of hydrolysis time $\left(t_{\mathrm{H}}\right) \cdot x_{\mathrm{H}}$ reaches an almost constant value of $33 \%$ after $120 \mathrm{~h}$ of etching.

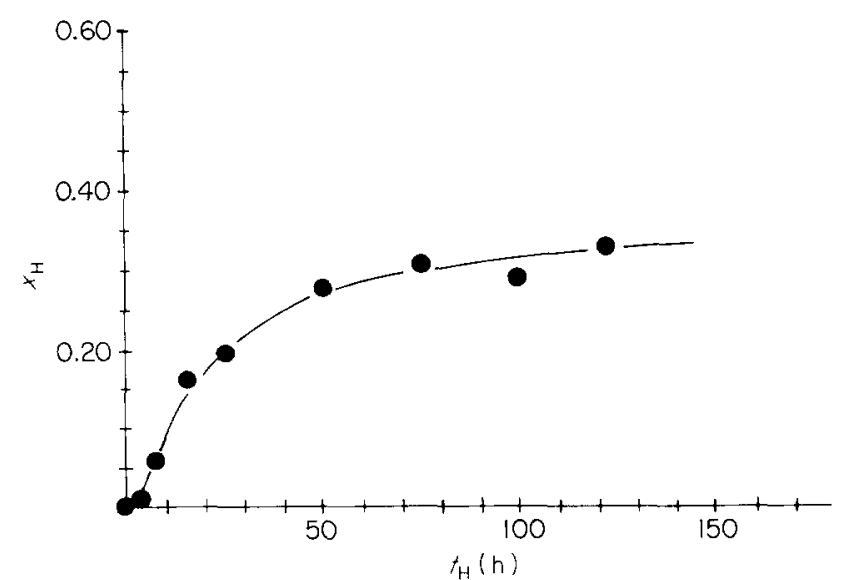

Figure 1 Fraction of material removed by hydrolysis etching $\left(x_{\mathrm{H}}\right)$ as a function of hydrolysis time $\left(t_{\mathrm{H}}\right)$

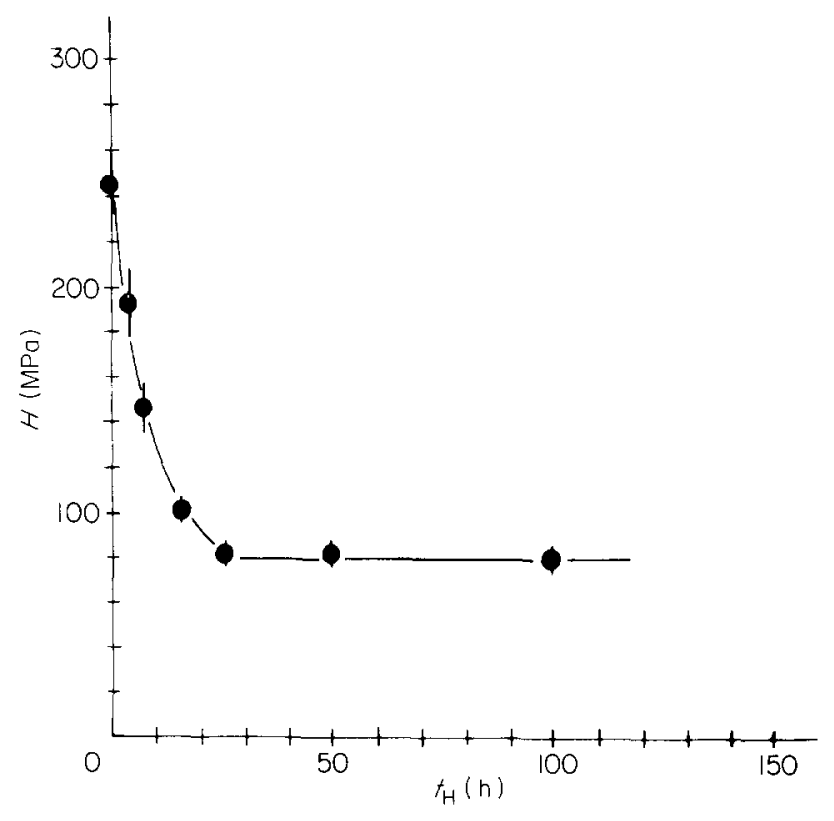

Figure 2 Microhardness $(H)$ as a function of hydrolysis time $\left(t_{\mathrm{H}}\right)$

Figure 2 shows $H$ as a function of $t_{\mathrm{H}}$. As can be seen, $H$ decreases drastically with increasing $t_{\mathbf{H}}$. It is interesting to note that a constant value of $H$ is reached after $25 \mathrm{~h}$, whereas the process of hydrolysis proceeds over a much longer period of time.

Figure 3 represents the WAXS diagram of the initial material and of three samples hydrolysed for different times. As can be seen, the intensity of the amorphous halo decreases and that of the crystal reflections increases with increasing $t_{\mathrm{H}}$. It is also noteworthy that even at the end of hydrolysis the sample remains partly amorphous. Furthermore, the half-peak width of the crystal reflections is not changed by the hydrolysis. This indicates that the average size of the crystals is not changed during the etching process.

The $x_{\mathrm{c}}$ values derived from the densities and from the WAXS diagrams are shown in Table 1 together with the measured densities. Before the hydrolysis treatment, one obtains $x_{c}=0.58$ from density measurements and $x_{\mathrm{c}}=0.56$ from WAXS. After hydrolysis, the corresponding values for $x_{\mathrm{c}}$ are 0.82 and 0.74 , respectively. In the case of the samples hydrolysed for 4,8 and $16 \mathrm{~h}$, the crystallinities as determined by density are far too small and also the scattering of the data was larger than usual. We attribute this to the presence of microvoids formed during hydrolysis.

Table $I$ also includes the molecular weights measured by viscometry. At the end of the hydrolysis process, a value of $M_{\mathrm{w}}=3200$ was found. This corresponds to 17 monomer units and to a length of the extended chain of, roughly, $18 \mathrm{~nm}$. One has to point out, however, that equation (1) is not proved to be strictly correct for such small $M_{\mathrm{w}}$ values. Therefore, the $M_{\mathrm{w}}$ values reported have to be taken as only approximate values.

\section{DISCUSSION}

\section{Comparison of WAXS and density measurements}

The values for $x_{c}$ obtained from density measurements differ from those derived from WAXS. While good agreement is obtained within experimental error for the sample which was not hydrolysed, the values obtained 

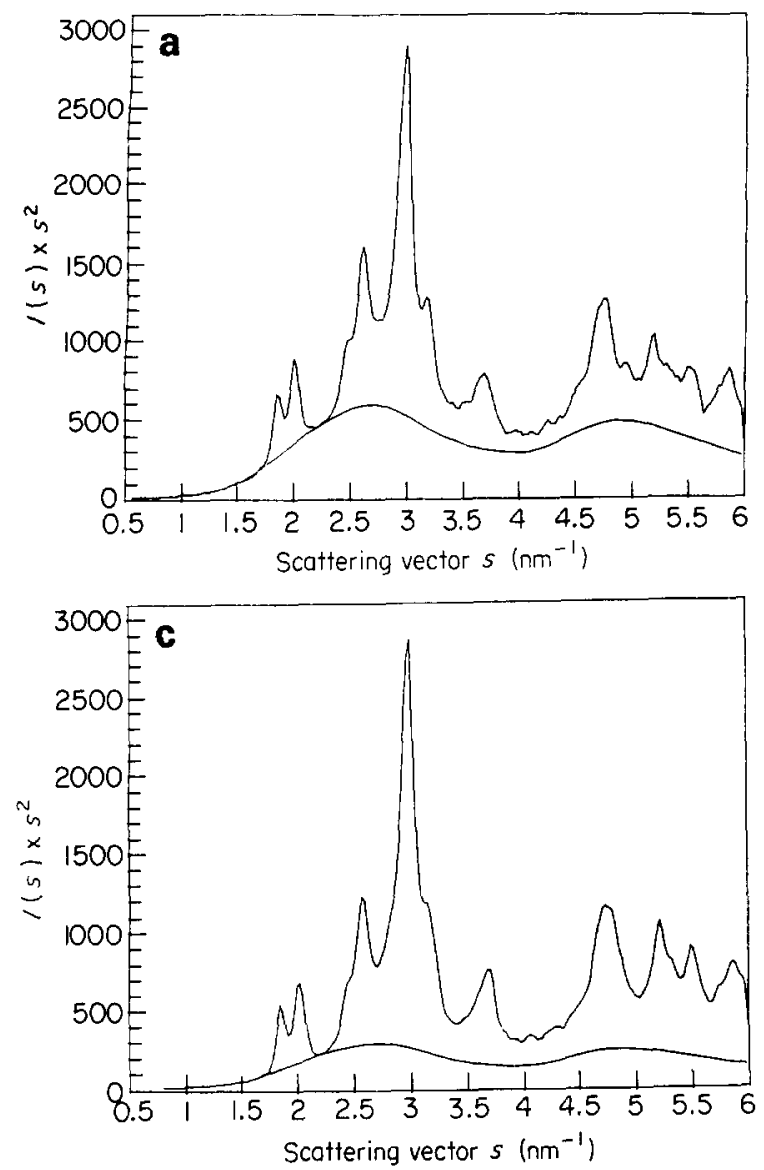
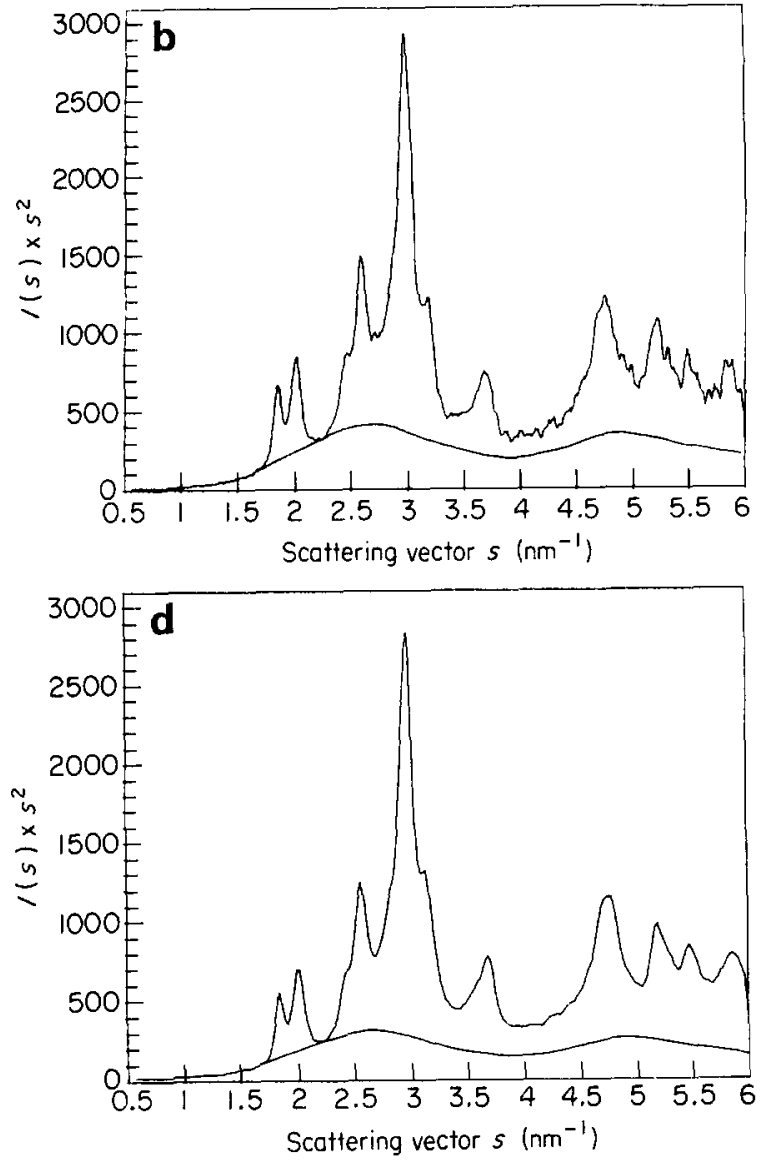

Figure 3 WAXS diagrams of samples hydrolysed for different times: (a) starting material; (b) $t_{\mathrm{H}}=4 \mathrm{~h}$; (c) $t_{\mathrm{H}}=50 \mathrm{~h}$; (d) $t_{\mathrm{H}}=122 \mathrm{~h}$

Table 1 Weight loss $\left(x_{\mathrm{H}}\right)$, molecular weight $\left(M_{\mathrm{w}}\right)$, density $(\rho)$ and crystallinity values $\left(x_{\mathrm{c}}\right)$ derived from density measurements and from WAXS for PET crystallized at $240^{\circ} \mathrm{C}$ for $24 \mathrm{~h}$ as a function of hydrolysis time $\left(t_{\mathrm{H}}\right)$

\begin{tabular}{rcclll}
\hline $\begin{array}{l}t_{\mathrm{H}} \\
(\mathrm{h})\end{array}$ & $\begin{array}{l}x_{\mathrm{H}} \\
(\%)\end{array}$ & $\begin{array}{l}M_{\mathrm{w}} \\
\left(\times 10^{-3}\right)\end{array}$ & $\begin{array}{l}\rho \\
\left(\mathrm{g} \mathrm{cm}^{-3}\right)\end{array}$ & $\begin{array}{l}x_{\mathrm{c}} \\
(\rho)\end{array}$ & $\begin{array}{l}x_{\mathrm{c}} \\
(\text { WAXS })\end{array}$ \\
\hline 0 & - & 45.5 & 1.421 & 0.58 & $0.56 \pm 0.03$ \\
4 & 1.0 & 12.6 & 1.411 & 0.52 & $0.68 \pm 0.03$ \\
8 & 6.1 & 9.9 & 1.368 & 0.23 & $0.65 \pm 0.03$ \\
16 & 16.4 & 5.5 & 1.355 & 0.14 & $0.72 \pm 0.03$ \\
26 & 19.3 & 5.6 & 1.457 & 0.80 & $0.69 \pm 0.03$ \\
50 & 27.8 & 4.8 & 1.459 & 0.82 & $0.74 \pm 0.03$ \\
75 & 31.2 & 3.2 & 1.458 & 0.81 & $0.63 \pm 0.03$ \\
100 & 28.8 & 3.2 & 1.456 & 0.80 & $0.73 \pm 0.03$ \\
122 & 33.2 & 3.2 & 1.459 & 0.82 & $0.74 \pm 0.03$ \\
\hline
\end{tabular}

from density of the samples hydrolysed for 4,8 and $16 \mathrm{~h}$ are definitely too small (Table 1). This effect has to be attributed to microvoid formation during removal of the hydrolysed material in agreement with results found on other oxidized polymer systems ${ }^{18}$.

In the case of the samples hydrolysed for $26 \mathrm{~h}$ and longer, the values obtained from density measurements were systematically larger than those from WAXS. From high values of density we conclude that in these samples, due to the larger extent of hydrolysis, the voids were accessible to the liquid in the density gradient column. That the values obtained from density were even larger than those obtained from WAXS may be caused by the fact that the value assumed for $\rho_{\mathrm{c}}$ in equation (2) is too small. The value of $x_{c}$ is more affected by an error in $\rho_{c}$ the larger is this quantity. If we assume for $\rho_{\mathrm{c}}$ the high value of $1.515 \mathrm{~g} \mathrm{~cm}^{-3}$ suggested by Huisman and Heuvel we obtain $x_{c}=0.72$, which is in good agreement with the value obtained by WAXS. On the other hand if we use the value of Huisman and Heuvel ${ }^{19}$ for calculating the crystallinity for the sample which is not hydrolysed we obtain $x_{\mathrm{c}}=0.51$ which is smaller than the $x_{\mathrm{c}}$ value obtained by WAXS. Therefore we propose that the high $\rho_{c}$ value of Huisman and Heuvel is only valid for the hydrolysed sample. It is reasonable to assume that the crystals formed by the short chains present after hydrolysis are more perfect and, therefore, have a higher density than those found in the high molecular weight semicrystalline material.

\section{Comparison of results with previous hydrolysis investigations}

The initial rate of weight loss in our experiment (up to $5 \mathrm{~h}$ etching time) is smaller than that found by Miyagi and Wunderlich ${ }^{6}$. This discrepancy seems to be due to the difference in the size of the polymer particles exposed to the water. In our case, the particles obtained by cutting the thin film were larger than in their experiment where a powder was used. We preferred to use a sliced thin film and cut it into small pieces because we did not want to risk changing the morphology by the stresses possibly induced through grinding during the preparation of the powder.

The weight loss curve shown in Figure $I$ as a function of $t_{\mathrm{H}}$ levels off at $\sim 32 \%$ while Miyagi and Wunderlich ${ }^{6}$ found limiting values up to $41 \%$. Though we repeated the experiment several times we did not obtain larger 
weight loss values. Such a difference must be, therefore, due either to the different material used (for example, a larger diethylene glycol content in the older samples used by the other authors) or to differences in the sample preparation. One should also note that the high value of $41 \%$ observed by Miyagi and Wunderlich is the result of just one measurement obtained on a single sample. In any event, due to the repetition of our experiment, we can be certain that for the sample investigated in the present study a limiting weight loss value of $32 \pm 2 \%$ was obtained.

For the next section it is important to discuss the value of $x_{c}$ at the end of the hydrolysis process. According to our WAXS measurements $x_{\mathrm{c}} \approx 0.72$, while Miyagi and Wunderlich claim to have found $x_{c}=1.00$. We believe, however, that the latter value is incorrect because of the values of $\rho_{\mathrm{c}}$ and $\rho_{\mathrm{a}}$ available at that time, namely $\rho_{\mathrm{c}}=1.445 \mathrm{~g} \mathrm{~cm}^{-3}$ and $\rho_{\mathrm{a}}=1.328 \mathrm{~g} \mathrm{~cm}^{-3}$ were too small and were corrected later to the values used in this paper (see Experimental section). If we use the new values ${ }^{11}$ of $\rho_{\mathrm{c}}=1.490 \mathrm{~g} \mathrm{~cm}^{-3}$ and $\rho_{\mathrm{a}}=1.334 \mathrm{~g} \mathrm{~cm}^{-3}$ we obtain for the samples of Miyagi and Wunderlich $x_{\mathrm{c}}=0.86$ which is closer to our results. We would also like to point out that some values of the density measured by Miyagi and Wunderlich were larger than the value of $\rho_{\mathrm{c}}$ assumed by these authors which was difficult to explain and which supports the larger value used in this paper. Taking all the foregoing evidence into consideration we conclude that in the samples of Miyagi and Wunderlich there exists

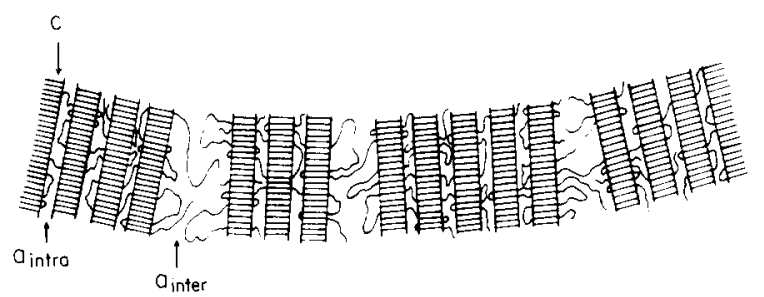

a
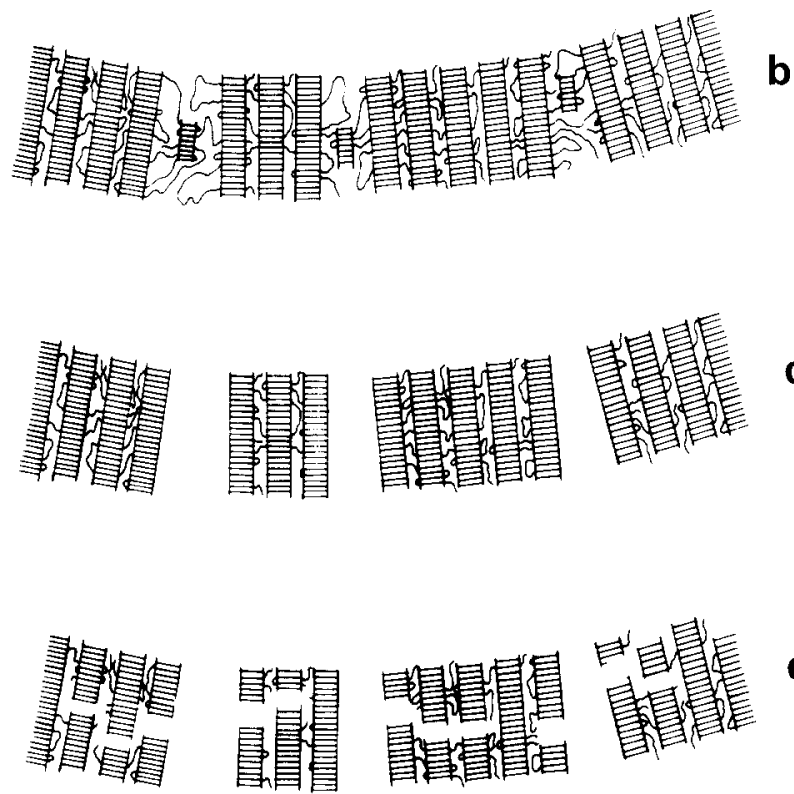

d

Figure 4 Schematic representation of the crystalline amorphous structure of PET at the different stages of etching: c, crystals; $a_{\text {intra }}$ intrastack amorphous regions; $a_{\text {inter }}$, interstack amorphous regions. For the sake of simplicity only a few chains have been indicated in the amorphous regions: (a) initial state; (b) end of the first stage $\left(t_{\mathrm{H}} \approx 4 \mathrm{~h}\right)$; (c) end of the second stage $\left(t_{\mathrm{H}} \approx 16 \mathrm{~h}\right) ;\left(\right.$ d) third stage $\left(t_{\mathrm{H}}>62 \mathrm{~h}\right)$ a considerable amount of amorphous material at the end of the etching process.

\section{Analysis of the weight loss caused by etching}

While the initial amorphous fraction of the sample is 0.45 according to WAXS and 0.42 according to density measurements, only a fraction of 0.33 is removed by hydrolysis. This means that, after hydrolysis is completed, the sample still contains amorphous regions. Obviously only a part of the amorphous material was removed. How can this be explained?

From recent SAXS results it was concluded ${ }^{12}$ that the amorphous regions in semicrystalline PET are not uniform in size. In fact, one has to distinguish (see Figure 4a) between:

1. amorphous layers between the crystals of a lamellar stack. These layers are uniform in thickness so that the scattering of the crystals within each stack is coherent. Each lamellar stack consists of about three to six crystal lamellae. The amorphous fraction within these stacks will be called the 'intrastack amorphous fraction', $x_{\mathrm{a}, \text { intra }}$;

2. amorphous regions separating different lamellar stacks. These regions are not uniform in size and are somewhat larger than the first ones. The distances between the lamellar stacks are randomly distributed so that they do not contribute to the angular position of the scattering maximum. The fraction of these regions will be called the interstack amorphous fraction', $x_{\mathrm{a} . \text { inter }}$.

Of course:

$$
x_{\mathrm{a}}=x_{\mathrm{a}, \text { inter }}+x_{\mathrm{a}, \text { intra }}
$$

Thus, if we denote the fraction of material within the lamellar stacks by $x_{\mathrm{L}}$, and the degree of crystallinity within the lamellar stacks by $x_{\mathrm{cL}}$, we can write for the overall degree of crystallinity:

Furthermore

$$
x_{\mathrm{c}}=x_{\mathrm{L}} x_{\mathrm{cL}}
$$

and

$$
\begin{gathered}
x_{\mathrm{a}, \text { inter }}=1-x_{\mathrm{L}} \\
x_{\mathrm{a}, \text { intra }}=x_{\mathrm{a}}-x_{\mathrm{a}, \text { inter }}
\end{gathered}
$$

In the case of the sample investigated in the present study, $x_{c}$, as measured by WAXS, is 0.56 and, according to our previous investigation ${ }^{12}, x_{\mathrm{cL}}=0.70$. Therefore, as a consequence of equation (4) the fraction of material inside of the lamellar stacks $x_{\mathrm{L}}$ is 0.80 and the amorphous fraction outside of the lamellar stacks $x_{\mathrm{a} \text {,inter }}\left(=1-x_{\mathrm{L}}\right)$ is 0.20 . This latter value is smaller than the total fraction of material removed by hydrolysis etching (0.32). Therefore, we conclude that by hydrolysis etching we totally remove the amorphous fraction between the lamellar stacks, and the additional part, $x_{\mathrm{H}}-x_{\mathrm{a}, \text { inter }}=0.10$, from the lamellar stacks themselves.

It is reasonable to assume that the etching of the material outside of the lamellar stacks proceeds more quickly than the etching of these stacks. In agreement with this assumption a change of slope of the curve in Figure $l$ is observed after $\sim 20 \%$ of the material is removed.

\section{Change of crystallinity during hydrolysis}

It is interesting to analyse the change of crystallinity in the course of hydrolysis. Already after $4 \mathrm{~h}$ when only 
$2 \%$ of the material has been removed, the crystallinity as measured by WAXS rises from 0.56 to 0.68 (see Table 1). This crystallinity increase clearly demonstrates that additional crystallization has occurred in the sample in the presence of water at $180^{\circ} \mathrm{C}$. This effect may be a consequence of the increased chain mobility in the presence of water and/or of the breaking of chain bonds which allow the molecules to assume conformations which favour crystallization. In any case we have to state that a considerable amount of crystallization occurs in the initial step of hydrolysis. As water was assumed to have penetrated only within the interstack amorphous regions at this stage of hydrolysis, we believe that these crystals are lying in the interstack regions as indicated in Figure $4 b$.

Let us now consider the next period (up to $16 \mathrm{~h}$ ) during which, according to our model described earlier, the interstack amorphous regions (which after the first hydrolysis stage contain some crystals) are removed. The crystallinity increases only a little from 0.68 to 0.72 (according to WAXS). At the same time $\sim 20 \%$ of the material has been removed by hydrolysis. This can be explained by the fact that the newly formed crystals within the interstack amorphous regions have also been removed. Because these new crystals are formed in the presence of water within the previous amorphous regions they are more strongly subjected to attack by water than the old crystals since water is unable to penetrate the old crystals.

The assumption that the newly formed crystals are removed is supported by a comparison of the measured and the calculated values of $x_{c}$ at a $t_{\mathrm{H}}$ of $16 \mathrm{~h}$. After removal of $x_{\mathrm{a}, \mathrm{inter}}$ (including the newly formed crystals) the expected degree of crystallinity is given by $x_{\mathrm{c}}=x_{\mathrm{cL}}$ which is 0.7 according to our previous publication ${ }^{12}$. This is in good agreement with the value measured by WAXS after $16 \mathrm{~h}$, namely 0.72 . Therefore the assumption that, after the second stage of the etching process, the interstack amorphous regions (including the nearly formed crystals) are removed is well supported by the crystallinity measurements. The resulting structure is schematically depicted in Figure 4c.

Let us, finally, consider the hydrolysis period of $16-120 \mathrm{~h}$ in which, according to our assumption, the lamellar stacks themselves are attacked. Although $\sim 12 \%$ of the material is removed during this last period, the degree of crystallinity is not changed within experimental error (see Table I). From this we have to conclude that not only amorphous regions but also crystalline regions are removed. Such an attack on the crystals obviously becomes possible after the water has penetrated the intrastack amorphous regions. It would be reasonable to assume that the removed crystals are those that are the thinnest or the most distorted. However in the WAXS patterns (see Figure 3) no sharpening of the crystalline reflections is observed.

In order to discuss this more quantitatively, we calculate the degree of crystallinity $\left(x_{\mathrm{ce}}\right)$ which we would expect at the end of hydrolysis if no fraction of the initial crystals is removed. Let us denote the initial fraction of crystals by $x_{\mathrm{co}}$ and the removed material at the end of hydrolysis by $x_{\mathrm{He}}$. If the fraction of crystals remains unchanged we can write:

$$
x_{\mathrm{ce}}=x_{\mathrm{co}} /\left(1-x_{\mathrm{He}}\right)
$$

where, according to Figure $1, x_{\mathrm{He}}$ is 0.32 . If we use the initial values obtained by WAXS $\left(x_{\mathrm{c}}=0.56\right)$ we obtain $x_{\text {ce }}=0.82$. As the measured value is 0.74 we have to conclude that $8 \%$ of the crystallized material was removed. If we use the density values, $x_{\mathrm{co}}$ is 0.58 and $x_{\mathrm{ce}}$ becomes 0.85 . The measured value, according to Table $l$, is 0.81 . According to this calculation, $4 \%$ of the crystallized material is removed instead of $8 \%$. To expect a better agreement would not be realistic in view of the experimental inaccuracy and the ambiguity in the value of $\rho_{\mathrm{c}}$ (see earlier) involved. Because of this ambiguity we trust the WAXS value more. Since the totally removed material during this final stage of hydrolysis is $\sim 12 \%$ we can conclude that more than half of the removed material was crystalline.

\section{Comparison of weight loss and microhardness}

Figure 2 shows that hydrolysis is accompanied by a considerable reduction of $H$. In a previous investigation ${ }^{18}$ a similar effect was observed with polyethylene and the decrease of $H$ was attributed to the formation of voids. The presence of voids is clearly demonstrated by density measurements in the case of the PET samples investigated in this study, too.

By comparison of Figures 1 and 2 it becomes obvious that the drastic decrease of $H$ stops after a $t_{\mathrm{H}}$ of $26 \mathrm{~h}$ where only $20 \%$ of the material has been removed. Further hydrolysis does not affect the $H$ value. As discussed before, we believe that the $20 \%$ is the fraction of interstack amorphous regions. Thus we conclude that the removal of the interstack amorphous regions leads to a decrease of $H$ whereas the further hydrolysis affecting the lamellar stacks leaves the hardness value unaffected. This may be so because no additional voids are introduced during this second process and also the crystalline fraction remains unaffected. Thus, our measurement of $H$ supports our interpretation in which hydrolysis can be separated into two different processes, the first one affecting the interstack regions, the other affecting the lamellar stacks.

\section{Molecular weight of the hydrolysed material}

In the completely hydrolysed material, a value of $M_{\mathrm{w}}=3200$ is obtained (Table 1). This value corresponds to a length of $18 \mathrm{~nm}$ for the completely extended chain. The average crystal thickness before hydrolysis was found to be $5.7 \mathrm{~nm}^{12}$. Thus each chain obtained after hydrolysis can in principle form up to three crystal stems. At this stage one may ask whether this result can be brought into agreement with the model presented in Figure 4.

Let us first assume that the breaking of the chemical bonds mainly takes place outside of the lamellar stacks. In this case, in order to obtain chain fragments of $18 \mathrm{~nm}$, some portions of each chain have to lie outside of the stacks and those parts which are situated within a lamellar stack, on average, must not exceed $16 \mathrm{~nm}$ in length. Are these chains long enough to build up the lamellar stack? Some possible chain trajectories in the case of a lamellar stack consisting of four crystal lamellae are depicted in Figure 5. As can be seen, the two inner crystal lamellae can be built up only by chains having at least a length of $30 \mathrm{~nm}$ (corresponding to $M_{\mathrm{w}}=6000$ ). However, the lamellae at the border of the stack can be built up by chains with a length of only $12 \mathrm{~nm}\left(M_{\mathrm{w}}=2400\right)$ or, if the chain end is located within the stack, $6 \mathrm{~nm}\left(M_{\mathrm{w}}=1200\right)$. This shows the following: it would be difficult to find agreement between our model and the molecular weight 


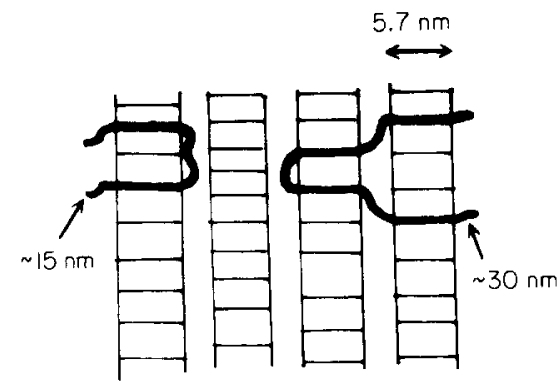

Figure 5 Schematic representation of two chain trajectories within a lamellar stack

measurements if it is assumed that the chemical bonds are broken only in the regions outside of the lamellar stacks. However, it is not impossible to obtain agreement, because there exists a distribution in chain lengths so that some parts may be considerably longer than $16 \mathrm{~nm}$ and, in addition, even the average value may be somewhat larger than $16 \mathrm{~nm}$ because of the ambiguity involved in applying equation (1) for the determination of the molecular weight.

An agreement between our model and the molecular weight measurement can be easily obtained if one takes into consideration that some chemical bonds are broken in the amorphous layers within the lamellar stacks. We want to point out that the breaking of a single chemical bond of a chain in the amorphous region is not sufficient to remove some material in the subsequent treatment with ethanol; at least two bonds have to be broken. According to our experimental results some material is removed from the amorphous layer inside each lamellar stack. Therefore we believe that some of the chemical bonds are also broken within the lamellar stacks.

Chains of lengths equal to multiples of the crystal thickness have been also found in etching experiments of polyethylene ${ }^{3}$. As pointed out ${ }^{3}$, the disappearance of these multiples of crystal thickness is clearly linked to the cutting of surface chain folds. With polyethylene the question of material not removed by dissolution does not have the relevance that it has with PET. Moreover in polyethylene there is no indication that amorphous regions outside of the lamellar stacks (denoted by $x_{\mathrm{a}, \text { inter }}$ ) exist.

Miyagi and Wunderlich ${ }^{6}$ claimed that the chain length corresponds to the crystal thickness. For this, they identified the crystal thickness with the long period as obtained from the maximum of the SAXS by applying Bragg's law. This procedure seems to us to be incorrect because $L$ represents the sum of the crystal thickness and the thickness of the amorphous region, and $L$ simply determined by applying Bragg's law is larger than the true value, as shown in a recent publication ${ }^{12}$. If one takes the above into consideration we can assume that, in the investigation by Miyagi and Wunderlich, the chains were longer than the thickness of the crystals.

\section{CONCLUSIONS}

By comparing the weight loss obtained by hydrolysis followed by ethanol treatment with results obtained by WAXS and by an elaborate evaluation of SAXS data, it is concluded that the etching process proceeds in three stages. In the first stage $(<4 \mathrm{~h})$, new crystals are formed within those amorphous regions which are lying between the semicrystalline lamellar stacks. In the second stage $(4-16 \mathrm{~h})$, the regions between the lamellar stacks are attacked and removed while the lamellar stacks themselves mainly remain unaffected. Finally, in the third stage ( $>25 \mathrm{~h}$ ), the lamellar stacks are attacked and part of both the crystalline and amorphous material regions in these stacks is removed. Thus etching by hydrolysis seems to be a powerful method to distinguish between amorphous regions which are differently located within the material.

\section{ACKNOWLEDGEMENTS}

We gratefully acknowledge CICYT, Spain for supporting this investigation (Grant MAT90-0795). HGZ thanks the Secretaria de Estado de Universidades e Investigación for the award of the Humboldt Mutis Prize. CV is indebted to the Government of Luxembourg for a grant.

\section{REFERENCES}

1 Wunderlich, B. 'Macromolecular Physics', Vol. 1, Academic Press, New York, 1973, p. 408

2 Palmer, R. P. and Cobbold, A. J. Makromol. Chem. 1964, 74, 174

3 Keller, A., Martuscelli, E., Priest, D. J. and Udagawa, Y. J. Polym. Sci., Polym. Phys. Edn 1971, 9, 1807

4 Battista, O. A., Coppick, S., Howsmon, J. A., Morehead, F. A. and Sisson, W. A. Ind. Eng. Chem. 1956, 48, 333

5 Koenig, J. L. and Agboatwalla, M. C. J. Macromol. Sci. 1968, B2, 391

6 Miyagi, A. and Wunderlich, B. J. Polym. Sci., Polym. Phys. Edn $1972,10,2072$

7 Miyagi, A. and Wunderlich, B. J. Polym. Sci., Polym. Phys. Edn $1972,10,2085$

8 Daubeny, R. P., Bunn, C. W. and Brown, C. J. Proc. R. Soc. A 1954, 531

9 Kilian, H. G., Halboth, H. and Jenckel, E. Kolloid Z. 1960, 172, 166

10 Konrad, G. and Zachmann, H. G. Kolloid Z. 1971, 247, 851

11 Gehrke, R. and Zachmann, H. G. Makromol. Chem. 1981, 182, 627

12 Santa Cruz, C., Stribeck, N., Zachmann, H. G. and Baltá Calleja, F. J. Macromolecules 1991, 24, 5980

13 Günther, B. and Zachmann, H. G. Polymer 1983, 24, 1008

14 Berkowitz, S. J. Appl. Polym. Sci. 1984, 29, 4353

15 Ruland, W. Acta Cryst. 1961, 14, 1180

16 Ruland, W. Polymer 1964, 5, 89

17 Baltá Calleja, F. J. Adv. Polym. Sci. 1985, 66, 117

18 Cagiao, M. E., Rueda, D. R. and Baltá Calleja, F. J. Colloid Polym. Sci. 1987, 265, 37

19 Huisman, R. and Heuvel, H. M. J. Appl. Polym. Sci. 1978, 22, 943 